\title{
Unsteady Simulations of Rocket Plume Expansions in Geostationary Earth Orbit
}

\author{
Andrew B. Weaver* and Iain D. Boyd ${ }^{\dagger}$ \\ University of Michigan, Ann Arbor, MI, 48109-2140, USA
}

\begin{abstract}
Unsteady, direct simulation Monte Carlo (DSMC)- particle-in-cell (PIC) simulations of rocket plume expansions into the magnetosphere at geostationary Earth orbit (GEO) are presented for three rocket thruster pulse durations: $0.1 \mathrm{~s}, 1.4 \mathrm{~s}$, and $9.9 \mathrm{~s}$. The chemical rocket thruster ejects hydrazine combustion products at a mass flow rate of $4.5 \times 10^{-4} \mathrm{~kg} / \mathrm{s}$, and the number densities are computed as a function of time for distances up to $35 \mathrm{~km}$ from the rocket thruster. The plume reaches steady-state operation $1 \mathrm{~km}$ downstream of the rocket thruster before the thruster is shut off at $1.4 \mathrm{~s}$, and steady-state operation is reached as far as $20 \mathrm{~km}$ downstream of the rocket thruster for the $9.9 \mathrm{~s}$ pulse duration. An analytic expression for free-molecular flow expanding into a vacuum is compared to the unsteady simulations for number densities, and the influences of energetic protons and electrons emitted from the Sun on predicted total number densities are investigated.
\end{abstract}

\section{Nomenclature}

$\dot{N}_{0, \mathrm{~s}} \quad$ Species number of particles ejected from the thruster exit per unit time $\left[\mathrm{s}^{-1}\right]$

$\vec{v} \quad$ Macroscopic velocity $\left[\mathrm{m} \mathrm{s}^{-1}\right]$

$d_{\text {ref }} \quad$ Reference diameter for VHS model $[\AA]$

$E_{\mathrm{b} \lambda} \quad$ Planck's spectral, black-body emissive power $\left[\mathrm{W} \mathrm{m}^{-2} \mu \mathrm{m}^{-1}\right]$

$k_{\mathrm{B}} \quad$ Boltzmann constant, $1.381 \times 10^{-23}\left[\mathrm{~J} \mathrm{~K}^{-1}\right]$

$M_{w} \quad$ Species molecular weight $\left[\mathrm{kg} \mathrm{kmol}^{-1}\right]$

$m_{\mathrm{s}} \quad$ Species mass $[\mathrm{kg}]$

$n_{\mathrm{e}} \quad$ Electron number density $\left[\mathrm{m}^{-3}\right]$

$n_{\mathrm{r}} \quad$ Index of refraction

$q_{0} \quad$ Elementary charge, $1.602 \times 10^{-19}[\mathrm{C}]$

$T_{0, \mathrm{~s}} \quad$ Species gas temperature at thruster exit $[\mathrm{K}]$

$T_{\mathrm{e}} \quad$ Electron temperature $[\mathrm{K}]$

$t_{\text {off }} \quad$ Time when thruster is shut off $[\mathrm{s}]$

$t_{\text {on }} \quad$ Time when thruster is turned on $[\mathrm{s}]$

$T_{\text {ref }} \quad$ Reference temperature for VHS model $[\mathrm{K}]$

$u \quad x$-component of velocity $\left[\mathrm{m} \mathrm{s}^{-1}\right]$

$U_{0} \quad$ Thruster exit velocity $\left[\mathrm{m} \mathrm{s}^{-1}\right]$

$w \quad z$-component of velocity $\left[\mathrm{m} \mathrm{s}^{-1}\right]$

Subscripts

$0 \quad$ Properties at thruster exit

ref Reference condition

s $\quad$ Species index

Symbols

*Postdoctoral Research Fellow, Department of Aerospace Engineering, 1320 Beal Ave., Ann Arbor, MI, and AIAA Senior Member

${ }^{\dagger}$ James E. Knott Professor of Engineering, Department of Aerospace Engineering, 1320 Beal Ave., Ann Arbor, MI, and AIAA Fellow 


$\begin{array}{ll}\beta & m_{\mathrm{s}} /\left(2 k_{\mathrm{B}} T_{0, \mathrm{~s}}\right)\left[\mathrm{s}^{2} \mathrm{~m}^{-2}\right] \\ \lambda & \text { Wavelength }[\mu \mathrm{m}] \\ \omega & \text { Viscosity index for VHS model } \\ \phi & \text { Electrostatic potential [V] }\end{array}$

\section{Introduction}

There are currently over 400 satellites in geostationary Earth orbit (GEO), ${ }^{1}$ and each satellite requires periodic firings of its rocket thrusters to maintain the proper orbit. GEO has a defined altitude of 35,786 $\mathrm{km}$ above sea level, which is well within the magnetosphere where high-energy particles emitted from the Sun may be found. Accurate predictive capabilities for rocket plume expansions in the magnetosphere are important in order to reduce risk of plume impingement on critical spacecraft surfaces.

The unsteady interaction between a rocket plume and the space environment at GEO is complex. Modeling the rocket plume expansion across tens of kilometers as a transient process involves: (1) gas expansion into a highly rarefied gas, (2) momentum and charge exchange collisions between protons emitted from the Sun and plume species, and (3) geomagnetic and electric field interactions with ions. The methodology for simulating such an environment is detailed by Stephani and Boyd. ${ }^{2,3}$ This methodology has also been applied to the Space Shuttle rocket plume expansion into the ionosphere to explain the correlation between rocket firings and measured ion fluxes aboard the international space station (ISS). ${ }^{4}$

Several others ${ }^{5-7}$ have also investigated the interactions between spacecraft, rocket plumes, and the space environment, but are focused on interactions at LEO. The lower densities, higher energies of ambient ions, and lower geomagnetic field strength at GEO relative to LEO changes the relative importance of the various plume-atmosphere interactions, and therefore a separate analysis is required to better understand and predict plume behavior at GEO. Recently, Stephani and Boyd ${ }^{3}$ studied spacecraft interactions with the magnetosphere with similar operating conditions and concluded that, unlike the ion plumes, neutral plumes were not strongly influenced by ionization mechanisms.

The aim of this work is to investigate the use of simplifying assumptions, such as no charge exchange collisions and free-molecular flow, for modeling time-dependent, neutral plume expansions from a rocket operated at GEO. Numerical simulations are carried out for three station-keeping maneuvers: $0.1 \mathrm{~s}, 1.4 \mathrm{~s}$, and $9.9 \mathrm{~s}$ pulse durations using a rocket thruster with a nominal mass flow rate of $4.5 \times 10^{-4} \mathrm{~kg} / \mathrm{s}$. Unsteady plume properties for the three station-keeping maneuvers are then compared to an analytic expression assuming free-molecular flow as a function of both spatial location and number flux at the thruster exit. The less limiting assumption of only momentum exchange collisions is applied to the same set of operating conditions and the neutral plume properties are quantitatively compared with the numerical solutions obtained without either assumption.

The remainder of the paper is organized a follows. Section II details the computational methods and collision models used to obtain numerical predictions of neutral plume properties. Analytic formulations for unsteady, free-molecular flow properties are provided in Section III, and time-dependent flow properties for the three station-keeping maneuvers obtained both numerically and analytically are presented in Section IV. Finally, concluding remarks are summarized in Section V.

\section{Modeling of Plume/Magnetosphere Interactions}

Modeling the plume interactions with the magnetosphere as it expands into the vacuum of space involves both rarefied and plasma gas dynamics. Therefore, a combination of two particle-based methods, direct simulation Monte Carlo ${ }^{8}$ (DSMC) and Particle-in-Cell ${ }^{9}$ (PIC), are employed simultaneously in the MONACO PIC $^{10}$ (MPIC) program used in this work. A description of the PIC-DSMC framework used in this work is provided in Section II.A, and the collision dynamics are described in Section II.B. The computational geometry and numerical parameters are described in Section II.C.

\section{II.A. PIC-DSMC Framework}

The DSMC method ${ }^{8}$ is used to model the motion and collisions of neutral particles, and the PIC $\operatorname{method}^{9}$ is used to model the charge exchange collisions (CEX) and ion acceleration due to electrostatic and magnetic fields. Bird's No Time Counter (NTC) method $^{8}$ is used to model the collision frequency, and particles are 
moved according to standard DSMC procedures. In the case of ions, an extra step is introduced before the particles are moved to compute the acceleration due to electrostatic and magnetic fields.

While standard PIC simulations ${ }^{9}$ model the electrons as particles, here the neutrals and ions are instead tracked with the electrons modeled as a fluid. Thus, the assumption of a Boltzmann energy distribution for the electron energy has been made; with the electrons equilibrated at a temperature $T_{e}$. This assumption greatly reduces the required computational resources since tracking electrons requires a much smaller time step than is used for ions. ${ }^{11}$ By further assuming assuming ideal, isothermal electron flow, quasi-neutrality, and negligible influence of the magnetic field on the electron momentum equation, the electrostatic potential may be related to the electron number density through the Boltzmann relation,

$$
\phi=\phi_{\mathrm{ref}}+\frac{k_{\mathrm{B}} T_{\mathrm{e}}}{q_{0}} \ln \left(\frac{n_{\mathrm{e}}}{n_{\mathrm{e}, \mathrm{ref}}}\right) .
$$

The electron number densities, $n_{e}$, are obtained in this PIC-DSMC framework from tracked ion number densities, $n_{i}$, since $n_{e} \approx n_{i}$ due to the quasi-neutrality assumption.

The acceleration due to the electric field is then obtained from the gradient of the electrostatic potential,

$$
\vec{E}=-\nabla \phi .
$$

The influence of the Earth's magnetic field at GEO on the ions is modeled using the magnetic field model described by Stephani and Boyd. ${ }^{2,12}$

\section{II.B. Collision Dynamics}

The hydrazine rocket thruster exhausts into high-energy electrons and $\mathrm{H}^{+}$ions originating from the Sun. Assuming complete combustion, the rocket thruster combustion forms the products: $\mathrm{H}_{2}, \mathrm{~N}_{2}$, and $\mathrm{NH}_{3}$. Therefore, there are three types of collisions considered in this work corresponding to momentum exchange between neutrals, momentum exchange between an ion and a neutral, and charge exchange. The redistribution of energy to internal energy modes of the colliding particles is ignored since the primary interest is in neutral number densities.

The momentum exchange between neutrals is modeled using the variable hard sphere (VHS) model, ${ }^{8}$ with the VHS model parameters listed in Table 1.

Table 1. VHS model parameters used for the plume neutral species

\begin{tabular}{|c|c|c|c|}
\hline Species & $T_{\text {ref }}[\mathrm{K}]$ & $d_{\text {ref }}[\AA]$ & $\omega$ \\
\hline $\mathrm{H}_{2}$ & 273 & 2.92 & 0.75 \\
$\mathrm{~N}_{2}$ & 273 & 4.17 & 0.75 \\
$\mathrm{NH}_{3}$ & 273 & 5.94 & 0.75 \\
\hline
\end{tabular}

Elastic collisions between ions and neutrals result in highly forward scattering. At the relatively high energies considered in this work $(1,000 \mathrm{eV})$ the scattering angles are low, ${ }^{13,14}$ and therefore it is assumed that all such collisions result in complete forward scattering.

The third type of collision considered in this work is charge exchange. These reactions are of the type: $\mathrm{A}^{+}+\mathrm{B} \longrightarrow \mathrm{A}+\mathrm{B}^{+}$, and if species $\mathrm{A}$ is different from species $\mathrm{B}$ then the charge exchange is nonresonant and involves an energy change. Most of the CEX collisions will require energy to proceed since the most frequent CEX collision is between the ambient $\mathrm{H}^{+}$ions and $\mathrm{H}_{2}$ from the plume. Cross sections for CEX collisions between $\mathrm{H}^{+}-\mathrm{H}_{2}$ and $\mathrm{H}^{+}-\mathrm{N}_{2}$ are obtained from Kusakabe et al. ${ }^{15}$ and Lindsay and Stebbings, ${ }^{16}$ respectively. No data has been found for the CEX cross section between $\mathrm{H}^{+}$and $\mathrm{NH}_{3}$, and therefore it is assumed to have the same cross section as $\mathrm{N}_{2}$. For the same reason, the scattering angles and direct transfer collision cross sections are assumed to all correspond to that of $\mathrm{H}^{+}-\mathrm{N}_{2}$ reported by Cabrera-Trujillo et al. ${ }^{14}$

Photons emitted from the Sun have sufficient energy to ionize neutral $\mathrm{H}_{2}$ molecules, but rarely collide to have much effect on the plume properties. The collision frequency may be computed by integrating the product of the solar black-body emissive power to photon energy ratio and the non-dissociative $\mathrm{H}_{2}$ 
photo-ionization cross section from Liu and Shemansky, ${ }^{17}$

$$
\begin{aligned}
\nu & =\int_{0}^{\infty} \frac{E_{\mathrm{b} \lambda}}{h c_{0} / \lambda} \sigma_{\mathrm{I}}\left(h c_{0} / \lambda\right) \mathrm{d} \lambda \\
& =\frac{2 \pi c_{0}}{n_{\mathrm{r}}^{2}} \int_{0}^{\infty} \frac{\sigma_{\mathrm{I}}\left(h c_{0} / \lambda\right)}{\lambda^{4}\left[\exp \left(\frac{h c_{0}}{n_{\mathrm{r}} \lambda k_{\mathrm{B}} T}\right)-1\right]} \mathrm{d} \lambda .
\end{aligned}
$$

The radiation is in the magnetosphere where the density is very low, and therefore a value of one is used for the index of refraction, $n_{\mathrm{r}}$, to calculate the non-dissociative $\mathrm{H}_{2}$ photo-ionization collision frequency of $1.8 \times 10^{-6} \mathrm{~s}^{-1}$. The non-dissociative photo-ionization process for $\mathrm{H}_{2}$ is more likely than dissociative, Compton, or double ionization, ${ }^{18}$ but its collision frequency of just $1.8 \times 10^{-6} \mathrm{~s}^{-1}$ is less than that of CEX collisions. Since the effect of CEX collisions on the neutral number density will be shown in Section IV.D to be less than $7 \%$, the effects of $\mathrm{H}_{2}$ photo-ionization would also be less and therefore are neglected.

\section{II.C. Computational Geometry and Numerical Parameters}

The plume flow is simulated in MPIC on a 2D, axisymmetric grid shown in Fig. 1 with the rocket thruster placed on the center of the axis of symmetry. The $0.9 \mathrm{~N}$ rocket thruster exhausts the combustion products: $\mathrm{H}_{2}, \mathrm{~N}_{2}$, and $\mathrm{NH}_{3}$ in the $+\mathrm{x}$-direction towards the outer computational boundary placed $35 \mathrm{~km}$ downstream. The geomagnetic field lines are oriented perpendicular to the thrust vector, or in the z-direction, and the magnetic field strength is reported by Chulliat et al. ${ }^{19}$ to be $1.1 \times 10^{-7} \mathrm{~T}$.

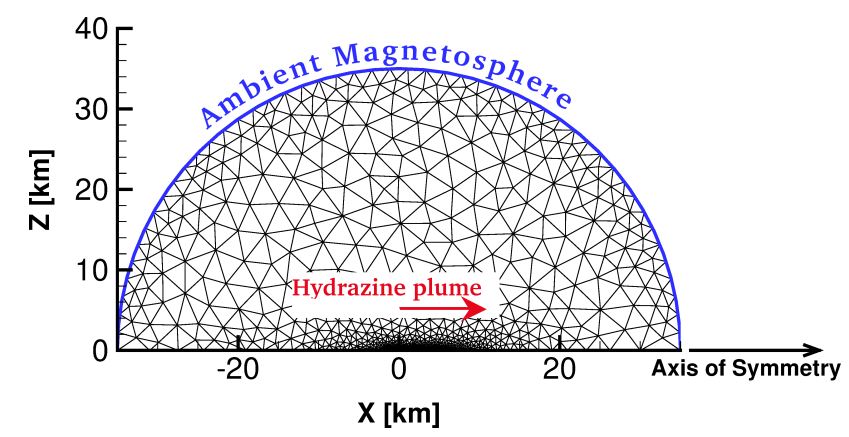

Figure 1. Computational geometry of $0.9 \mathrm{~N}$ rocket thruster at GEO

A total of four ambient $\mathrm{H}^{+}$ions are randomly positioned in each cell with energies sampled from the Boltzmann distribution at $1 \mathrm{keV}$. In order to maintain the ambient number density of $3 \times 10^{6} \mathrm{~m}^{-3}$, the particle weights are allowed to vary according to cell size. These ambient particles are not moved and do not change velocity or charge as a result of momentum or charge exchange collisions, respectively. These procedures are employed in order to ensure a sufficient number of collisions with the ambient $\mathrm{H}^{+}$ions occur in this low-density environment. Although the assumption that $\mathrm{H}^{+}$ions do not deplete is weak near the thruster where the number of $\mathrm{H}^{+}-\mathrm{H}_{2}$ collisions is the highest, this assumption allows for a worst-case scenario for the prediction of neutral plume properties. The properties of the plume species at the rocket exhaust and the ambient ions are detailed in Table 2.

Table 2. Ambient and rocket nozzle exhaust conditions

\begin{tabular}{|c|c|c|c|c|c|}
\hline Species & $M_{w}\left[\mathrm{~kg} \mathrm{kmol}^{-1}\right]$ & $k_{\mathrm{B}} T / q_{0}[\mathrm{eV}]$ & $U_{0}[\mathrm{~km} / \mathrm{s}]$ & $n\left[\mathrm{~m}^{-3}\right]$ & $\dot{N}_{0, \mathrm{~s}}\left[\mathrm{~s}^{-1}\right]$ \\
\hline $\mathrm{H}^{+}$ & 1.01 & $1.00 \times 10^{3}$ & 0 & $3.00 \times 10^{6}$ & 0 \\
$\mathrm{H}_{2}$ & 2.02 & 0.03 & 1.91 & $2.42 \times 10^{23}$ & $1.24 \times 10^{22}$ \\
$\mathrm{~N}_{2}$ & 28.0 & 0.03 & 1.91 & $1.35 \times 10^{22}$ & $6.82 \times 10^{21}$ \\
$\mathrm{NH}_{3}$ & 17.0 & 0.03 & 1.91 & $5.79 \times 10^{22}$ & $2.92 \times 10^{21}$ \\
\hline
\end{tabular}

In Table $2, M_{w}$ is the species molecular weight, $U_{0}$ is the thruster exit velocity, and $\dot{N}_{0, \mathrm{~s}}$ is the species number of particles ejected from the thruster exit per unit time.

Samples are averaged over 10,000 time steps in order to obtain an unsteady solution of the plume expansion into the low-density $\mathrm{H}^{+}$flow. The time step is 10 microseconds, and therefore the averages correspond to a $0.1 \mathrm{~s}$ interval. 


\section{Unsteady, Free-Molecular Flow from a Point Source}

Exact, analytic solutions exist for flows in the limit of free-molecular flow and no external forces. In literature ${ }^{8}$ flows are classified as free-molecular if the ratio of mean free path to characteristic length, $\lambda / L$, is greater than 10. This ratio is known as the Knudsen number, or Kn, and for the plume expansion flow in consideration the Knudsen number increases from about $71 \mathrm{~km}$ downstream of the thruster to over 1,000 $35 \mathrm{~km}$ downstream of the thruster. Thus, it is worthwhile to formulate an analytic expression for number densities in an unsteady, free-molecular flow which may be used for comparison with MPIC simulations.

The formulation for the unsteady, free-molecular flow in the absence of external forces begins with the Boltzmann equation for a single species, ${ }^{8,20}$

$$
\frac{\partial f}{\partial t}+\vec{v} \cdot \frac{\partial f}{\partial \vec{x}}+\vec{F} \cdot \frac{\partial f}{\partial \vec{v}}=Q(\vec{x}, t ; \vec{v})+J(\vec{x}, t) .
$$

Assuming no external forces and no collisions, the third term on the left hand side and the collision operator term, $J(\vec{x}, t)$, on the right hand side are zero. The source term, $Q(\vec{x}, t ; \vec{v})$, is introduced to account for the flux of particles ejected from the thruster exit. The formulation follows from Narasimha, ${ }^{20}$ where the velocity distribution function may be expressed as

$$
f(\vec{x}, t ; \vec{v})=f_{0}(\vec{x} ; \vec{v})+\int_{0}^{t} Q[\vec{x}-\vec{v}(t-s), s ; \vec{v}] \mathrm{d} s,
$$

and $f_{0}$ is the initial velocity distribution function at time $t=0 \mathrm{~s}$. For the case of no flow prior to the start of the station-keeping maneuver, $f_{0}$ is zero everywhere.

At large distances from the thruster such that the distance is much greater than the radius of the thruster exit, the flow would appear to be originating from a point. Therefore, a point-source flow may be assumed by representing the source flow as a product of dirac delta functions and the biased Maxwellian velocity distribution function,

$$
Q(\vec{x}, t ; \vec{v})=\delta(\vec{x}) \dot{N}_{0}(t) K u(\beta / \pi)^{3 / 2} \exp \left[-\beta\left\{\left(u-U_{0}\right)^{2}+v^{2}+w^{2}\right\}\right] .
$$

The normalization constant, $K$, in Eq. 6 is obtained from integration of the velocity distribution function over all velocity space,

$$
\begin{aligned}
K & =\left\{\int_{-\infty}^{\infty} \int_{-\infty}^{\infty} \int_{0}^{\infty} u \exp \left[-\beta\left(\left(u-U_{0}\right)^{2}+v^{2}+w^{2}\right)\right] \mathrm{d} u \mathrm{~d} v \mathrm{~d} w\right\}^{-1} \\
& =\frac{2 \beta}{\pi}\left\{\sqrt{\frac{\pi}{\beta}} U_{0}\left(1+\operatorname{erf}\left[\sqrt{\beta} U_{0}\right]\right)+\frac{\exp \left[-\beta U_{0}^{2}\right]}{\beta}\right\}^{-1},
\end{aligned}
$$

where it has been assumed that the mean thruster exit velocity is only in the $+\mathrm{x}$-direction with a magnitude equal to $U_{0}$. Moments of the distribution function such as number density and velocity may be obtained through integration over all velocity space,

$$
\begin{aligned}
& n(\vec{x}, t)=\int_{-\infty}^{\infty} \int_{-\infty}^{\infty} \int_{0}^{\infty} f(\vec{x}, t ; \vec{v}) \mathrm{d} u \mathrm{~d} v \mathrm{~d} w \\
& \vec{v}(\vec{x}, t)=\frac{1}{n(\vec{x}, t)} \int_{-\infty}^{\infty} \int_{-\infty}^{\infty} \int_{0}^{\infty} \vec{v} f(\vec{x}, t ; \vec{v}) \mathrm{d} u \mathrm{~d} v \mathrm{~d} w .
\end{aligned}
$$

Assuming that the flow is instantaneously turned on and off, as is assumed in the MPIC simulations, the time-dependent number of particles introduced at the thruster exit may be expressed as a product of the heaviside step function, $\dot{N}_{0, \mathrm{~s}}(t)=\dot{N}_{0, \mathrm{~s}} H(t)$. This greatly simplifies the integrations in Eq. 8 and Eq. 9 such 
that the number density may now be expressed as

$$
\begin{aligned}
n_{\mathrm{on}, \mathrm{s}}(\vec{x}, t) & =H\left(t-t_{\mathrm{on}}\right) \frac{K \dot{N}_{0, \mathrm{~s}} x}{4 \beta^{3 / 2}\left(x^{2}+z^{2}\right)^{5 / 2}} \exp \left(-\beta U_{0}^{2}\right)\left\{\sqrt{\pi}\left(x^{2}\left(2 \beta U_{0}^{2}+1\right)+z^{2}\right)\right. \\
& \times \exp \left[\beta \frac{x^{2} U_{0}^{2}}{x^{2}+z^{2}}\right] \operatorname{erfc}\left[\frac{\sqrt{\beta}\left(\frac{x^{2}+z^{2}}{t-t_{\mathrm{on}}}-x U_{0}\right)}{\sqrt{x^{2}+z^{2}}}\right] 2 \sqrt{\beta\left(x^{2}+z^{2}\right)}\left(\frac{x^{2}+z^{2}}{t-t_{\mathrm{on}}}+x U_{0}\right) \\
& \left.\times \exp \left[-\beta \frac{x^{2}+z^{2}}{\left(t-t_{\mathrm{on}}\right)^{2}}-\frac{2 x U_{0}}{t-t_{\mathrm{on}}}\right]\right\} .
\end{aligned}
$$

A pulse may be modeled by adding

$$
\begin{aligned}
n_{\mathrm{off}, \mathrm{s}}(\vec{x}, t) & =-H\left(t-t_{\mathrm{off}}\right) \frac{K \dot{N}_{0, \mathrm{~s}} x}{4 \beta^{3 / 2}\left(x^{2}+z^{2}\right)^{5 / 2}} \exp \left(-\beta U_{0}^{2}\right)\left\{\sqrt{\pi}\left(x^{2}\left(2 \beta U_{0}^{2}+1\right)+z^{2}\right)\right. \\
& \times \exp \left[\beta \frac{x^{2} U_{0}^{2}}{x^{2}+z^{2}}\right] \operatorname{erfc}\left[\frac{\sqrt{\beta}\left(\frac{x^{2}+z^{2}}{t-t_{\mathrm{off}}}-x U_{0}\right)}{\sqrt{x^{2}+z^{2}}}\right] 2 \sqrt{\beta\left(x^{2}+z^{2}\right)}\left(\frac{x^{2}+z^{2}}{t-t_{\mathrm{off}}}+x U_{0}\right) \\
& \left.\times \exp \left[-\beta \frac{x^{2}+z^{2}}{\left(t-t_{\mathrm{off}}\right)^{2}}-\frac{2 x U_{0}}{t-t_{\mathrm{off}}}\right]\right\} .
\end{aligned}
$$

to the number density in Eq. 10 after the thruster is shut off at time $t=t_{\text {off }}$. Multiple pulses may also be modeled in this manner by expressing the species number density as a summation of all $M$ pulses

$$
n_{\mathrm{s}}(\vec{x}, t)=\sum_{i}^{M}\left(n_{\mathrm{on}, \mathrm{s}, \mathrm{i}}(\vec{x}, t)+n_{\mathrm{off}, \mathrm{s}, \mathrm{i}}(\vec{x}, t)\right)
$$

The total number density for the entire plume is then equal to the sum of all the species number densities computed from Eq. 12,

$$
n(\vec{x}, t)=\sum_{\mathrm{s}} n_{\mathrm{s}}(\vec{x}, t) .
$$

Velocities are similarly obtained from the integral in Eq. 9,

$$
\begin{aligned}
u_{\mathrm{on}, \mathrm{s}}(\vec{x}, t) & =H\left(t-t_{\mathrm{on}}\right) \frac{K \dot{N}_{0, \mathrm{~s}} x^{2}}{4 n_{\mathrm{on}, \mathrm{s}}(\vec{x}, t) \beta^{2}\left(x^{2}+z^{2}\right)^{7 / 2}} \exp \left(-\beta U_{0}^{2}\right)\left\{\sqrt{\pi \beta} x U_{0}\left(x^{2}\left(2 \beta U_{0}^{2}+3\right)+3 z^{2}\right)\right. \\
& \times \exp \left[\beta \frac{x^{2} U_{0}^{2}}{x^{2}+z^{2}}\right] \operatorname{erfc}\left[\frac{\sqrt{\beta}\left(\frac{x^{2}+z^{2}}{t-t_{\mathrm{on}}}-x U_{0}\right)}{\sqrt{x^{2}+z^{2}}}\right] 2 C \sqrt{x^{2}+z^{2}} \\
& \left.\times \exp \left[-\beta \frac{x^{2}+z^{2}}{\left(t-t_{\mathrm{on}}\right)^{2}}-\frac{2 x U_{0}}{t-t_{\mathrm{on}}}\right]\right\} \\
w_{\mathrm{on}, \mathrm{s}}(\vec{x}, t) & =H\left(t-t_{\mathrm{on}}\right) \frac{K \dot{N}_{0, \mathrm{~s}} x z}{4 n_{\mathrm{on}, \mathrm{s}}(\vec{x}, t) \beta^{2}\left(x^{2}+z^{2}\right)^{7 / 2}} \exp \left(-\beta U_{0}^{2}\right)\left\{\sqrt{\pi \beta} x U_{0}\left(x^{2}\left(2 \beta U_{0}^{2}+3\right)+3 z^{2}\right)\right. \\
& \times \exp \left[\beta \frac{x^{2} U_{0}^{2}}{x^{2}+z^{2}}\right] \operatorname{erfc}\left[\frac{\sqrt{\beta}\left(\frac{x^{2}+z^{2}}{t-t_{\mathrm{on}}}-x U_{0}\right)}{\sqrt{x^{2}+z^{2}}}\right] 2 C \sqrt{x^{2}+z^{2}} \\
& \left.\times \exp \left[-\beta \frac{x^{2}+z^{2}}{\left(t-t_{\mathrm{on}}\right)^{2}}-\frac{2 x U_{0}}{t-t_{\mathrm{on}}}\right]\right\} \\
& =u_{\mathrm{on}, \mathrm{s}} \frac{z}{x},
\end{aligned}
$$


with the factor, $C$, defined as

$$
C=\beta\left[\frac{x^{4}+z^{4}+2 x^{2} z^{2}}{\left(t-t_{\mathrm{on}}\right)^{2}}+U_{0} \frac{x^{3}+x^{2}}{t-t_{\mathrm{on}}}+x^{2} U_{0}^{2}\right] .
$$

The velocities after the thruster is shut off are simply the opposites of the velocities during the pulse in Eqs. 14 and 15, with time $t_{\text {on }}$ replaced with $t_{\text {off }}$. Thus, the velocities may be obtained for each species as a summation of all $M$ pulses,

$$
\vec{v}_{\mathrm{s}}(\vec{x}, t)=\sum_{i}^{M}\left(\vec{v}_{\mathrm{on}, \mathrm{s}}(\vec{x}, t)+\vec{v}_{\mathrm{off}, \mathrm{s}}(\vec{x}, t)\right)
$$

\section{Results}

The $0.9 \mathrm{~N}$ rocket thruster is initialized at time $t=t_{\mathrm{on}}=0 \mathrm{~s}$ with the conditions listed in Table 2, and the flow field is the ambient $\mathrm{H}^{+}$flow. The rocket thruster is then switched off at times $t_{\text {off }}=0.1 \mathrm{~s}, t_{\text {off }}=1.4$ $\mathrm{s}$ and $t_{\mathrm{off}}=9.9 \mathrm{~s}$. Total number densities are computed at $0.1 \mathrm{~s}$ intervals and extracted at locations: $(1 \mathrm{~km}$, $\left.10^{\circ}\right),\left(5 \mathrm{~km}, 10^{\circ}\right),\left(10 \mathrm{~km}, 10^{\circ}\right)$, and $\left(20 \mathrm{~km}, 10^{\circ}\right)$ downstream of the rocket thruster.

IV.A. Single Pulse, $t_{\text {off }}=0.1 \mathrm{~s}$

A peak in total number density is observable from Fig. 2 at a location $1 \mathrm{~km}$ downstream of the thruster, while two peaks may be observed at the $5 \mathrm{~km}$ location. These peak values are just $23 \%$ and $6 \%$ of the steady-state values for the $1 \mathrm{~km}$ and $5 \mathrm{~km}$ locations, respectively. Therefore, there is insufficient time to reach steady-state operation at any of the four locations for the $0.1 \mathrm{~s}$ pulse.

The existence of multiple peaks in Fig. 2 for the $5 \mathrm{~km}$ location is due to differences in species mass. The lighter $\mathrm{H}_{2}$ molecules travel at greater speeds than either the $\mathrm{N}_{2}$ or $\mathrm{NH}_{3}$ molecules, and therefore reach the $5 \mathrm{~km}$ location first and results in the first observed peak in number density. The second peak in number density is due to the arrival of the slower $\mathrm{N}_{2}$ and $\mathrm{NH}_{3}$ molecules.

At both locations, the analytic solution to the number density in Eq. (13) is in good agreement with the MPIC simulations - agreement to within $5 \%$ of the peak number density. Two peaks are also observable in the analytic solution at the $5 \mathrm{~km}$ location, but the analytic solution predicts a slower decay after the thruster is shut off. This deviation after the thruster is shut off will be seen for all station-keeping maneuvers considered in this work, and is due to the assumption of a free-molecular flow.
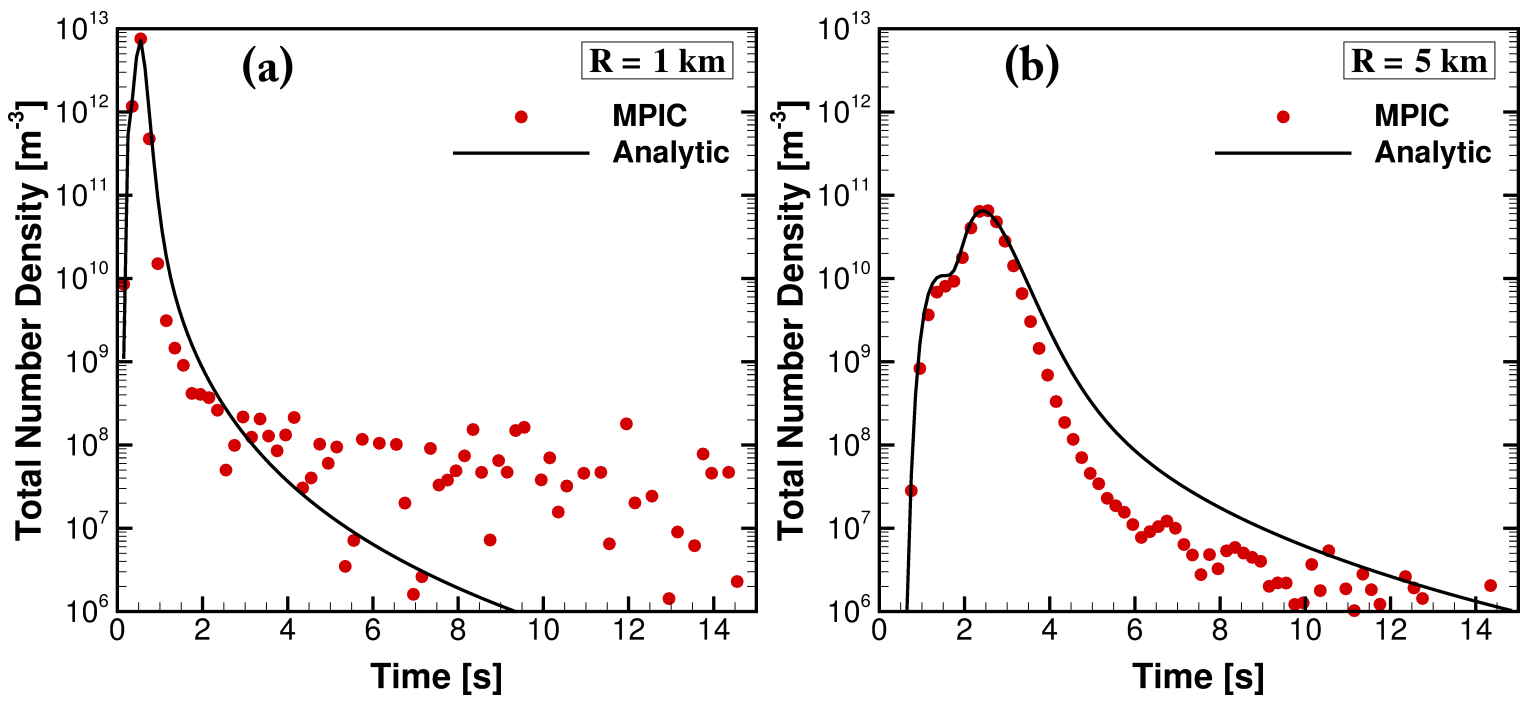

Figure 2. Total number densities (a) $1 \mathrm{~km}, 10^{\circ}$ and (b) $5 \mathrm{~km}, 10^{\circ}$ downstream of the rocket thruster corresponding to a $t_{\text {off }}=0.1$ s pulse maneuver. Symbols: MPIC with $k_{\mathrm{B}} T_{\mathrm{e}} / q_{0}=1,000 \mathrm{eV}$; solid line: analytic solution from Eq. 13 . 
IV.B. Single Pulse, $t_{\mathrm{off}}=1.4 \mathrm{~s}$

Increasing the pulse duration from $0.1 \mathrm{~s}$ to $1.4 \mathrm{~s}$ allows sufficient time for the plume to reach steady-state operation $1 \mathrm{~km}$ downstream of the thruster. The peak number density is observed in Fig. 3 to plateau at $1 \mathrm{~km}$, but is $73 \%$ of the steady-state value at the $5 \mathrm{~km}$ location. Again, the analytic solution is in good agreement at both locations until the thruster is shut off - at which point the effects of molecular collisions on number densities are more noticeable. The peak number density predicted by the analytic solution is within $8 \%$ of the value from MPIC.
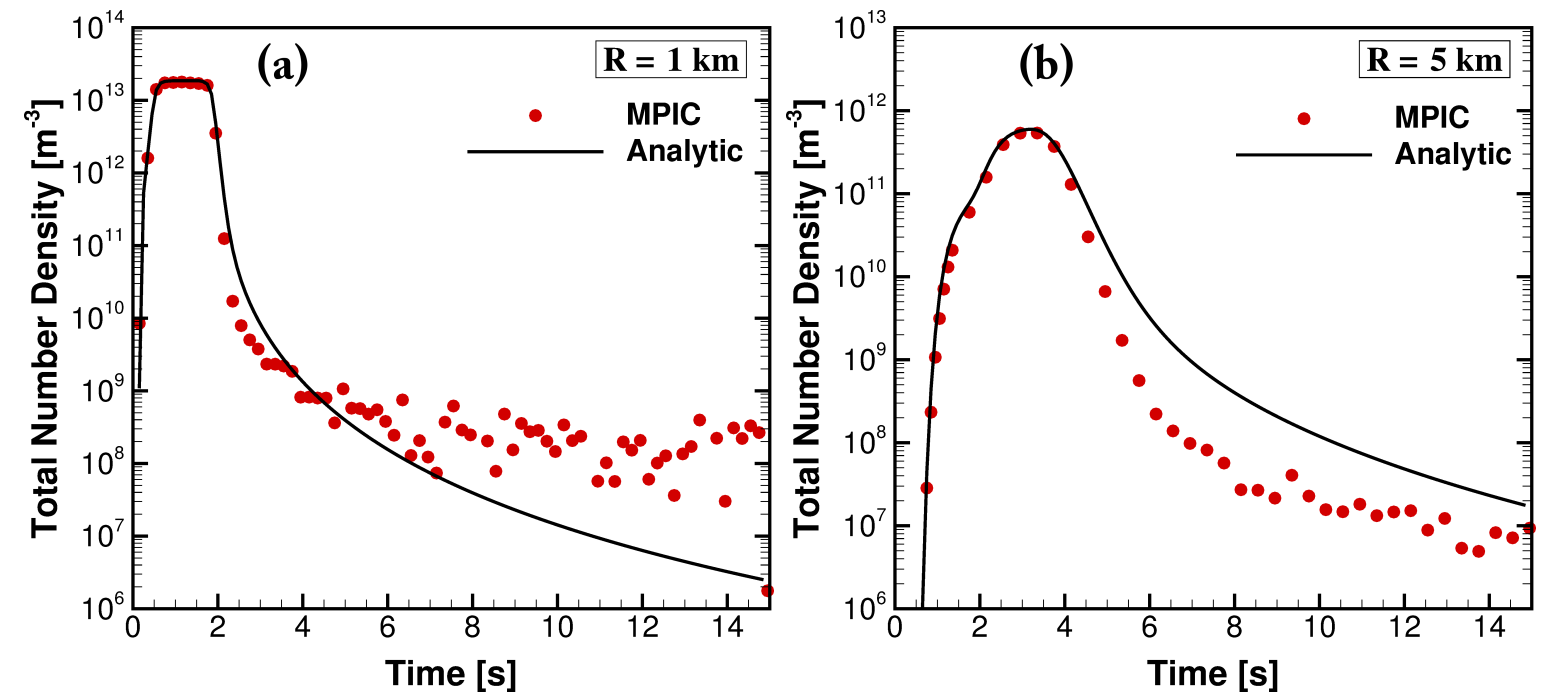

Figure 3. Total number densities (a) $1 \mathrm{~km}, 10^{\circ}$ and (b) $5 \mathrm{~km}, 10^{\circ}$ downstream of the rocket thruster corresponding to a $t_{\text {off }}=1.4 \mathrm{~s}$ pulse maneuver. Symbols: MPIC with $k_{\mathrm{B}} T_{\mathrm{e}} / q_{0}=1,000 \mathrm{eV}$; solid line: analytic solution from Eq. 13 .

IV.C. Single Pulse, $t_{\text {off }}=9.9 \mathrm{~s}$

The total number densities corresponding to electron/ion energies equilibrated at 1,000 eV and with no CEX collisions are illustrated in Fig. 4. It is easily observable from Fig. 4 that the total number density plateaus before the thruster is shut off, and this indicates that the total number density has reached steady-state values. The total number densities at the farthest probed location of $20 \mathrm{~km}$ from the thruster exit is shown in Fig. 4 to approach steady state, but does not plateau as observed at the closer locations.

The plume propagates at a finite rate, and therefore as the probed location is moved farther away from the rocket thruster the number density plateau is shifted to later times. Also, the peak total number densities reduce by orders of magnitude as distance from the rocket thruster is increased due to diffusion. The total number density is more than $10^{13} \mathrm{~m}^{-3} 1 \mathrm{~km}$ from the thruster, but is just $3 \times 10^{10} \mathrm{~m}^{-3}$ at $20 \mathrm{~km}$.

The total number density predictions using Eq. 13 are observed in Fig. 4 to be in good agreement with the MPIC simulations. The analytic peak number density is within $30 \%$ of the MPIC simulations with $k_{\mathrm{B}} T_{\mathrm{e}} / q_{0}=1,000 \mathrm{eV}$ for the $1 \mathrm{~km}, 5 \mathrm{~km}, 10 \mathrm{~km}$, and $20 \mathrm{~km}$ locations. This difference is due to the neglect of collisions in the analytic expression, and therefore the agreement with numerical solutions would improve with lower pressures at the thruster exit.

Agreement between the analytic solutions from Eq. 12 and from MPIC simulations also differs for each species. The agreement is best for the heaviest species, $\mathrm{N}_{2}$, while the worst agreement is for the lightest species, $\mathrm{H}_{2}$. The primary interest in this work is the neutral plume as a whole, but comparing each individual neutral species in the plume reveals that the peak $\mathrm{H}_{2}$ number densities tend to be as much as twice those predicted from MPIC. Peak number densities for $\mathrm{N}_{2}$, on the other hand, agree to within $30 \%$ of those from MPIC - on the same level of agreement as the overall neutral plume.

\section{IV.D. Influence of CEX Collisions on Neutral Plume}

Based on the reported electron and ion flux spectra measurements made over several solar cycles at GEO, ${ }^{21}$ the electron and ion energy distributions are found to be far from equilibrium. Thomsen et al. ${ }^{21}$ have also reported the $95 \%$ confidence interval (CI) for the flux spectra; which were then used to set the $95 \%$ CI for 

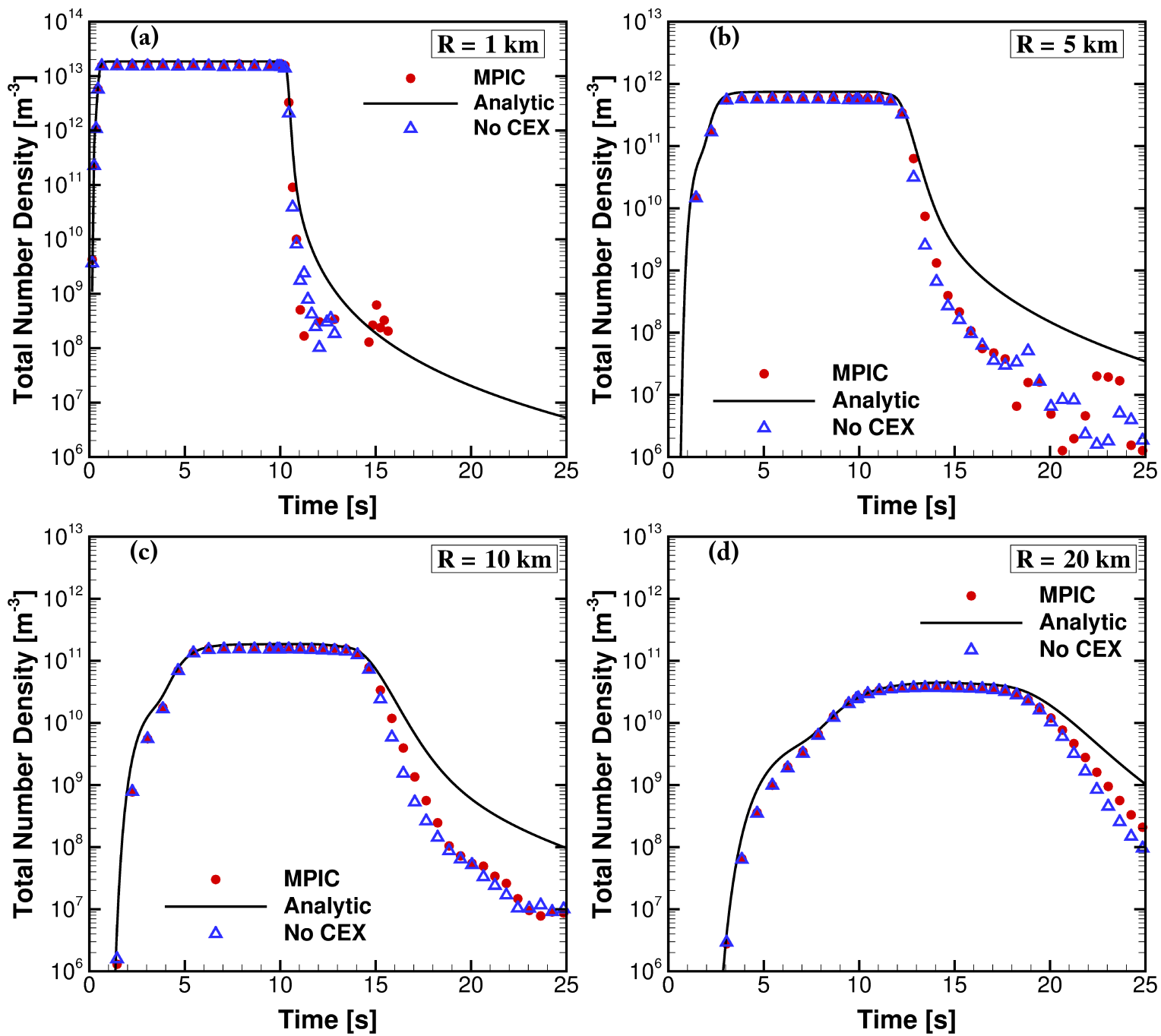

Figure 4. Total number densities (a) $1 \mathrm{~km}, 10^{\circ}$; (b) $5 \mathrm{~km}, 10^{\circ}$; (c) $10 \mathrm{~km}, 10^{\circ}$; and (d) (b) $20 \mathrm{~km}, 10^{\circ}$ downstream of the rocket thruster corresponding to a $t_{\mathrm{off}}=9.9 \mathrm{~s}$ pulse maneuver. Symbols: $\mathrm{MPIC}$ with $k_{\mathrm{B}} T_{\mathrm{e}} / q_{0}=1,000 \mathrm{eV}$; solid line: analytic solution from Eq. 13.

energy distributions as depicted as shaded regions in Fig. 5. The $t_{\text {off }}=9.9 \mathrm{~s}$ simulations were repeated with CEX collisions being neglected.

It may be observed from Fig. 4 that the electron/ion energy energies have little influence on the peak total number densities. Up to $20 \mathrm{~km}$ downstream of the rocket thruster, the negligible ambient electron/ion energy results in less than $7 \%$ lower total number densities. Deviations between the two cases tend to increase shortly after the thruster is shut off, but at which point the number densities rapidly decrease to near-ambient conditions. This result is useful in that it not only places a lower bound on expected number densities originating from uncertainties in ambient electron/ion energies, but also helps validate the use of analytic expressions for free-molecular flow with no external forces into a vacuum. ${ }^{20,22}$

\section{Conclusions}

Neutral number densities have been obtained for a range of station-keeping maneuvers and compared with unsteady, free-molecular flow solutions. For each maneuver, the analytic solution is in good agreement with the MPIC simulations. More noticeable differences observed between the two solutions after the thruster is shut off is due to the assumption of a free-molecular flow in the analytic solution.

Collisional effects are the most pronounced for the lightest, neutral species, $\mathrm{H}_{2}$. Peak number densities for $\mathrm{H}_{2}$ at each location predicted using the analytic expression are about twice the values predicted using 

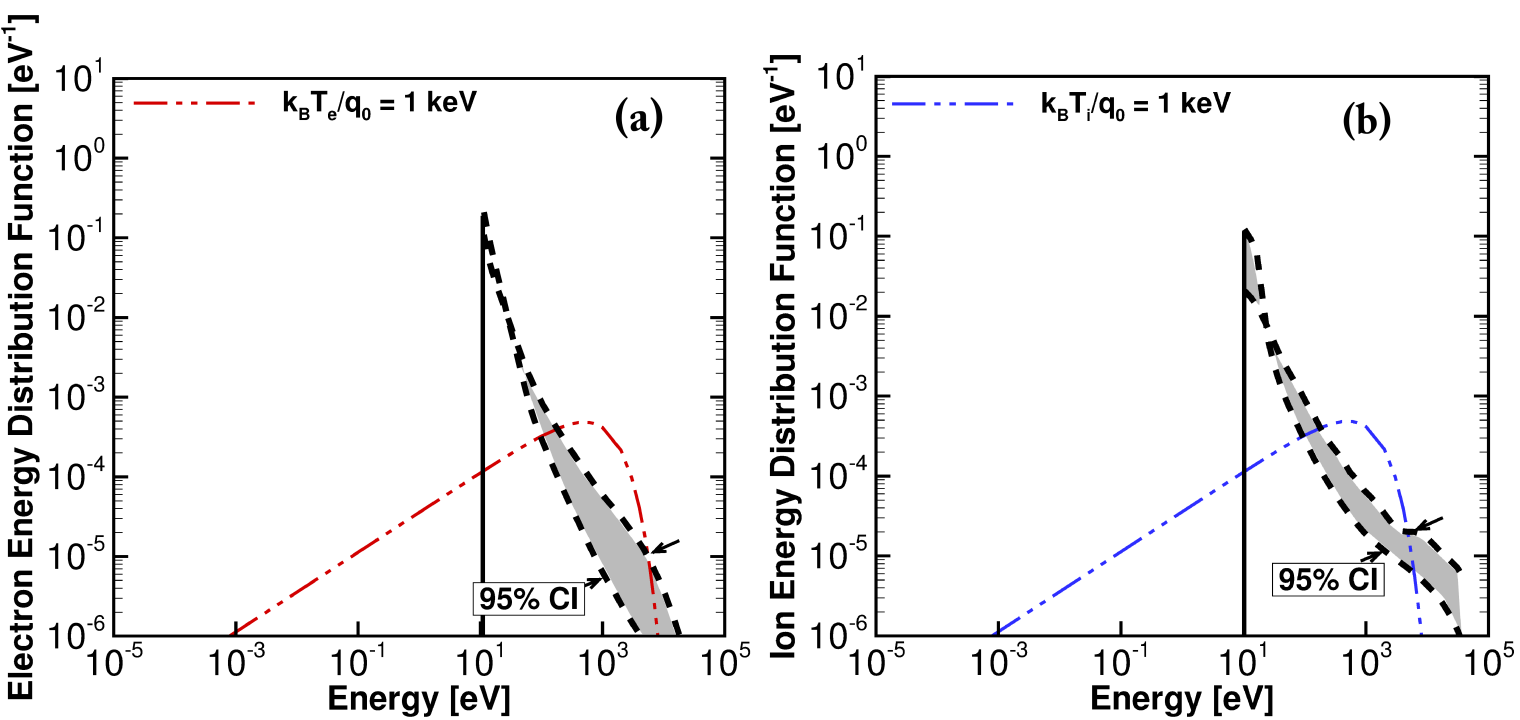

Figure 5. (a) Electron and (b) ion energy distribution functions derived from electron and ion flux spectra by Thomsen et al. ${ }^{2}$

MPIC. This difference is larger than the 15-30\% difference calculated for the heavier, $\mathrm{N}_{2}$ species.

Other effects, including CEX collisions and non-dissociative $\mathrm{H}_{2}$ photo-ionization, have been determined to be negligible for neutral number density predictions. Although assuming ambient $\mathrm{H}^{+}$ions do not deplete is questionable, especially near the thruster, it provides a worst-case scenario for its effects on neutral number densities. The result is a mere $7 \%$ lower, peak neutral number density prediction. Molecular hydrogen is the most prevalent species, and since the photo-ionization collision frequency is less than that of CEX collisions, photo-ionization at GEO is expected to have negligible effects on neutral plume properties.

\section{Acknowledgments}

The support of Air Force Research Laboratory (AFRL) Space Vehicles Directorate and Barron Associates, Inc. for this work under subcontract FA9453-15-C-0002 is gratefully acknowledged.

\section{References}

${ }^{1}$ Kelso, T. S., "NORAD Two-Line Element Sets Current Data," http://www.celestrak.com/noRAD/elements/, Accessed: 2015-09-28

${ }^{2}$ Stephani, K. A. and Boyd, I. D., "Detailed modeling and analysis of spacecraft plume/ionosphere interactions in low Earth orbit," J. Geophys. Res. Sp. Phys., Vol. 119, No. 3, 2014, pp. 2101-2116.

${ }^{3}$ Stephani, K. A. and Boyd, I. D., "Spacecraft plume interactions with the magnetosphere plasma environment in geostationary Earth orbit," J. Geophys. Res. Sp. Phys., Vol. 121, No. 2, feb 2016, pp. 1402-1412.

${ }^{4}$ Stephani, K. A., Boyd, I. D., Balthazor, R. L., McHarg, M. G., Mueller, B. A., and Adams, R. J., "Analysis and observation of spacecraft plume / ionosphere interactions during maneuvers of the space shuttle," J. Geophys. Res. Sp. Phys., Vol. 119, 2014, pp. 7636-7648.

${ }^{5}$ Mendillo, M., "Modification of the ionosphere by large space vehicles," Adv. Sp. Res., Vol. 2, No. 3, 1982, pp. $150-159$.

${ }^{6}$ Murad, E., "Spacecraft Interaction with Atmospheric Species in Low Earth Orbit," J. Spacecr. Rockets, Vol. 33, No. 1, 1996, pp. 131-136.

${ }^{7}$ Zhong, J., Zeifman, M. I., Levin, D. A., and Gimelshein, S. F., "Direct Simulation Monte Carlo Modeling of Homogenous Condensation in Supersonic Plumes," AIAA J., Vol. 43, No. 8, 2005, pp. 1784-1796. 1994.

${ }^{8}$ Bird, G. A., Molecular Gas Dynamics and the Direct Simulation of Gas Flows, Oxford University Press, New York,

${ }^{9}$ Birdsall, C. K. and Langdon, a. B., Plasma Physics via Computer Simulation, IOP Publishing Ltd, New York, 1991.

${ }^{10}$ Cai, C., Theoretical and Numerical Studies of Plume Flows in Vacuum Chambers, Ph.D. thesis, University of Michigan, Ann Arbor, 2005.

${ }^{11}$ Serikov, V. V., Kawamoto, S., and Nanbu, K., "Particle-in-cell plus direct simulation Monte Carlo (PIC-DSMC) approach for self-consistent plasma-gas simulations," IEEE Trans. Plasma Sci., Vol. 27, No. 5, 1999, pp. 1389-1398.

${ }^{12}$ Stephani, K. A. and Boyd, I. D., "Dynamics of Spacecraft Plume / Magnetosphere Interactions in Geostationary Earth Orbit," 53rd AIAA Aerosp. Sci. Meet., AIAA Paper 2015-1396, Kissimmee, FL, Jan. 5-7 2015, pp. 1-13. 
${ }^{13}$ Krstić, P. S. and Schultz, D. R., "Elastic and vibrationally inelastic slow collisions : $\mathrm{H}+\mathrm{H}_{2}, \mathrm{H}^{+}+\mathrm{H}_{2}$," J. Phys. B At. Mol. Phys., Vol. 32, 1999, pp. 2415-2431.

${ }^{14}$ Cabrera-Trujillo, R., Öhrn, Y., Deumens, E., Sabin, J., and Lindsay, B., "Theoretical and experimental studies of the $\mathrm{H}^{+}-\mathrm{N}_{2}$ system: Differential cross sections for direct and charge-transfer scattering at kilo-electron-volt energies," Phys. Rev. A, Vol. 66, No. 4, 2002, pp. 1-7.

${ }^{15}$ Kusakabe, T., Asahina, K., Gu, J. P., Hirsch, G., Buenker, R. J., Kimura, M., Tawara, H., and Nakai, Y., "Chargetransfer processes in collisions of $\mathrm{H}^{+}$ions with $\mathrm{H}_{2}, \mathrm{D}_{2}, \mathrm{CO}$, and $\mathrm{CO}_{2}$ molecules in the energy range 0.2-4.0 keV," Phys. Rev. A, Vol. 62, No. 6, 2000, pp. 62714 .

${ }^{16}$ Lindsay, B. G. and Stebbings, R. F., "Charge transfer cross sections for energetic neutral atom data analysis," J. Geophys. Res. Sp. Phys., Vol. 110, No. A12213, 2005, pp. 1-10.

${ }^{17}$ Liu, X. and Shemansky, D. E., "Nondissociative electron and photon ionization cross sections of molecular hydrogen and deuterium," J. Phys. B At. Mol. Opt. Phys., Vol. 45, No. 9, 2012, pp. 095203.

${ }^{18}$ Yan, M., Sadeghpour, H. R., and Dalgarno, A., "Photoionization Cross Sections of He and H2," Astrophys. J., Vol. 496, No. 2, 1998, pp. 1044-1050.

${ }^{19}$ Chulliat, A., Macmillan, S., Alken, P., Beggan, C., Nair, M., Hamilton, B., Woods, A., Ridley, V., Maus, S., and Thomson, A., "The US / UK World Magnetic Model for 2015-2020," Tech. rep., National Geophysical Data Center, NOAA, 2015.

${ }^{20}$ Narasimha, R., "Collisionless expansion of gases into vacuum," J. Fluid Mech., Vol. 12, No. 2, 1962, pp. $294-308$.

${ }^{21}$ Thomsen, M. F., Denton, M. H., Lavraud, B., and Bodeau, M., "Statistics of plasma fluxes at geosynchronous orbit over more than a full solar cycle," Sp. Weather, Vol. 5, No. S03004, 2007, pp. 1-9.

${ }^{22}$ Cai, C. and Boyd, I., "Theoretical and Numerical Study of Free Molecular-Flow Problems," J. Spacecr. Rockets, Vol. 44, No. 3, 2007, pp. 619-624. 